\title{
Arzneimitteltherapie in der Gastroenterologie - Stand und Perspektiven
}

Ende 2002, vor fast 20 Jahren, hat die PaulMartini-Stiftung zuletzt ihr Herbstsymposium der Gastroenterologie gewidmet. Höchste Zeit also, einen Überblick zu geben, wo wir heute mit der Arzneimitteltherapie in diesem medizinischen Fachgebiet stehen und wohin es gehen könnte. Das ist aus mehreren Gründen gerechtfertigt: Gastrointestinale Erkrankungen sind weit verbreitet und folgen nach den HerzKreislauf-Krankheiten in Deutschland auf Platz 2. Jährlich werden 2,5 Millionen Patient:innen mit Krankheiten der Verdauungsorgane stationär in Kliniken behandelt, mit Diagnosen von Bauchspeicheldrüsenentzündungen über chronischentzündliche Darmerkrankungen bis zu Magen- und Speiseröhrenkrebs. Und rund 60000 Menschen sterben jedes Jahr an Krankheiten der Verdauungsorgane. Dabei deckt die Gastroenterologie gleichermaßen die drei großen Ätiologien ab: entzündliche, infektiöse und maligne Erkrankungen.

Das erwartet Sie: der Gastrointestinaltrakt als Schlüsselsystem für die Pharmakotherapie; die Therapie chronisch entzündlicher Darmerkrankungen auf dem Weg in die nächste Dekade mit neuen molekularen Grundlagen, Therapiestrategien und Zielstrukturen; neue Entwicklungen in der Hepatologie im Bereich Hepatitiden, Steatohepatitis und Leberzellkarzi- nom. Und schließlich stellen unsere Vortragenden im Rahmen ihrer jeweiligen „Vision 2030“ Zellmigration als Target, Forschungsschwerpunkte der Industrie und zukünftige Strategien gegen gastrointestinale Tumoren vor.

\section{Interessenkonflikt}

Prof. Dr. Britta Siegmund has served as consultant for AbbVie, Arena, BMS, Boehringer Ingelheim, Celgene, Falk, Galapagos, Janssen, Lilly, Pfizer, Prometheus and Takeda and received speaker's fees from AbbVie, CED Service GmbH, Falk, Ferring, Janssen, Novartis, Pfizer, Takeda [served as representative of the Charité].

Prof. Dr. Stefan Endres hat eine Lizenzvereinbarung mit TCR2 (Biotechfirma, Cambridge, USA).

\section{Autoren}

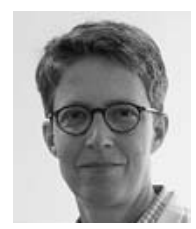

\section{Britta Siegmund}

Prof. Dr. med., Medizinische Klinik für Gastroenterologie, Infektiologie, Rheumatologie (einschl. Ernährungsmedizin), Campus Benjamin Franklin, Charité - Universitätsmedizin Berlin (CBF)

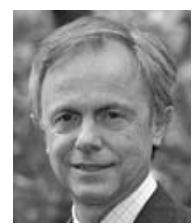

\section{Stefan Endres}

Prof. Dr. med., Abteilung für Klinische Pharmakologie, Medizinische Klinik und Poliklinik IV, Klinikum der Ludwig-Maximilians-Universität München

\section{Korrespondenzadressen}

Prof. Dr. med. Britta Siegmund Medizinische Klinik für Gastroenterologie, Infektiologie, Rheumatologie (einschl. Ernährungsmedizin) Campus Benjamin Franklin Charité - Universitätsmedizin Berlin (CBF) Hindenburgdamm 30

12203 Berlin

Deutschland

britta.siegmund@charite.de

Prof. Dr. med. Stefan Endres

Abteilung für Klinische Pharmakologie Medizinische Klinik und Poliklinik IV

Klinikum der Ludwig-Maximilians-Universität München

Lindwurmstraße 2a

80337 München

Deutschland

endres@Imu.de 